\title{
PROPOSTA DE PRODUTO ASSISTIVO PARA PESSOAS COM PARALISIA EM PUNHO E MÃO
}

Gisele Bortolaz Guedes - (gisele@bortolaz.com.br) - PPGEM, Universidade Tecnológica Federal do Paraná

Lucas Barboza Zattar Paganin (paganin@alunos.utfpr.edu.br) - PPGEM, Universidade Tecnológica Federal do Paraná

Milton Borsato (borsato@utfpr.edu.br) - PPGEM, Universidade Tecnológica Federal do Paraná

José Aguiomar Foggiatto (foggiatto@utfpr.edu.br) - PPGEM, Universidade Tecnológica Federal do Paraná

Fernando Henrique Fernandes (nandohfernandes@gmail.com) - Universidade Federal do Rio Grande do Norte

\section{RESUMO}

O domínio das habilidades motoras é essencial para o alcance da autonomia de um indivíduo. Pessoas que apresentam algum tipo de deficiência podem utilizar produtos de tecnologia assistiva para atender suas necessidades particulares, minimizando as dificuldades decorrentes da respectiva incapacidade. A tecnologia assistiva é um conjunto de recursos classificados de acordo com a necessidade funcional do usuário, abrangendo produtos como os de auxílio da vida diária, órteses e próteses. O produto final proposto por esta pesquisa é caracterizado como um objeto para auxiliar as atividades diárias daqueles que possuem deficiência oriunda da lesão do plexo braquial e que, apresentam alguma paralisia do punho e mão. O objetivo é desenvolver um produto de fácil adaptação e que possua mais de uma utilidade aos pacientes, proporcionando-lhes uma maior autonomia diante de suas limitações. Além disso, este trabalho apresentará uma comparação entre o processo de desenvolvimento de produtos tradicionais e produtos de tecnologia assistiva. A partir de informações obtidas na literatura e de entrevistas realizadas com pessoas que apresentam as limitações supracitadas, foi definido um produto que atenda às suas necessidades, o qual foi modelado e apresentado na forma de um mockup produzido em PLA por impressão $3 D$ para posterior validação dos usuários.

Palavras-chave: Produtos de Tecnologia Assistiva. Processo de Desenvolvimento de Produto. Paralisia em Punho e Mão. Impressão 3D.

Área: $O$ design e o desenvolvimento de produtos e serviços

\section{INTRODUÇÃO}

O uso de tecnologias assistivas pode ser considerado uma importante estratégia de reabilitação para auxiliar nas atividades funcionais de pessoas que apresentem alguma deficiência motora (ØIEN, FALLANG e ØSTENSJØ, 2015). De acordo com a Organização Mundial da Saúde da ONU, podem ser considerados produtos de tecnologia assistiva qualquer produto, instrumento, equipamento ou tecnologia adaptado ou especialmente elaborado para a melhoria funcional de pessoas com alguma deficiência. Dessa forma, como exemplo de tais produtos, pode-se citar dispositivos de mobilidade, braile, aplicativos de smartphone que auxiliem pessoas com deficiências audiovisuais, entre outros. Assim, de acordo com Borg, Larsson e Östergren (2011), as Tecnologias Assistivas (TA) podem ser usadas diretamente em 
alguma estrutura corporal para a melhoria de suas funções, com o intuito de proporcionar uma maior participação nas suas atividades.

De acordo com Paula, Faria e Vieira (2007) a atividade motora é fundamental para a formação e para o desenvolvimento do ser humano. Nos casos em que ocorre paralisia do punho e da mão, a incapacidade de realizar atividades diárias básicas como, por exemplo, o ato de se alimentar, de fazer a higienização pessoal e de realizar a limpeza de casa, torna esses pacientes demasiadamente dependentes de outros indivíduos. O desenvolvimento de uma TA que apoie esses indivíduos na realização de suas atividades diárias permite assim, o alcance da autonomia, a retomada da autoconfiança e a reinserção social.

Conforme Peng, Heim e Mallick (2014), a colaboração do usuário final é de grande importância para o alcance de sucesso no Processo de Desenvolvimento de Produtos (PDP). Assim, uma participação efetiva dos usuários dos produtos de tecnologia assistiva nas fases conceituais e de design fará com que o produto final atenda às expectativas do indivíduo em relação aos fatores de uso, ergonômicos e visuais. Além disso, o uso de uma tecnologia de manufatura aditiva (impressão 3D) é essencial para visualização do produto final obtido, o qual deverá ser validado com os respectivos usuários em pesquisas posteriores.

Dessa maneira, o objetivo principal deste artigo é apresentar uma proposta de produto assistivo versátil para pessoas com paralisia em punho e mão que proporcione a melhoria da qualidade de vida dos pacientes frente às suas limitações, e que facilite a realização de atividades básicas como a alimentação e higienização. Paralelamente, será caracterizado o Processo de Desenvolvimento de Produtos Assistivos a fim de realizar uma comparação entre o PDP para produtos tradicionais e para produtos de TA.

Para tanto, este artigo está estruturado da seguinte maneira: no primeiro capítulo foi apresentada a introdução ao assunto a ser discutido. Nos capítulos 2, 3 e 4 serão apresentados o referencial teórico acerca dos assuntos abordados neste artigo. Os aspectos metodológicos são apresentados no capítulo 5. Por fim, os capítulos 6 e 7 expõem respectivamente os resultados e as considerações finais.

\section{O PROCESSO DE DESENVOLVIMENTO DE PRODUTO}

Krishnan e Ulrich (2001) afirmam que o desenvolvimento de produtos é a transformação de uma oportunidade de mercado em um produto para venda, através de um processo que envolve recursos humanos, financeiros e tecnológicos. Buss e Cunha (2002) afirmam que este processo, em sua essência, é interdisciplinar e interfuncional. Interdisciplinar, porque depende da aplicação de conhecimentos de diversas áreas. Interfuncional porque todas as áreas funcionais da empresa devem se envolver, em maior ou menor grau no processo.

O processo de desenvolvimento de produtos (PDP) é descrito por inúmeros autores - Asimov (1962), Back (1983), Pahl e Beitz (2003), Pahl et al. (2005), Rozenfeld et al. (2006), Back et al. (2008), entre outros - por meio de modelos que representam as atividades, os métodos, as técnicas e as ferramentas para o projeto em engenharia.

De forma geral, estes modelos apresentam semelhanças em suas fases e etapas. Desta forma, é possível agrupar o processo de desenvolvimento de produtos nas seguintes fases e etapas:

- Fase de Pré-Desenvolvimento do Produto, a qual busca alinhar o planejamento estratégico da empresa e o planejamento estratégico do produto e, consequentemente, o planejamento do projeto;

- Desenvolvimento do Produto, o qual envolve a definição do problema de projeto, ciclo de vida, usuários e necessidades do projeto, projeto informacional (especificação de 
problemas de projeto), projeto conceitual (síntese de soluções alternativas para o produto), projeto preliminar (configuração do leiaute do produto, dimensionamento, modelagem, simulação de sistemas técnicos ou de tecnologias), projeto detalhado (normatização, representação e documentação de sistemas técnicos);

- Pós-desenvolvimento do produto, que contempla o acompanhamento do processo e do produto no mercado, retirada e descarte do produto.

O modelo a ser utilizado neste trabalho é o proposto por Rozenfeld, et al. (2006), o qual é composto por três fases: Pré-desenvolvimento, Desenvolvimento e Pós desenvolvimento, como pode ser visto na Figura 1.

Figura 1: Etapas do Processo de Desenvolvimento de Produtos. Fonte: Rozenfeld et al. (2006).

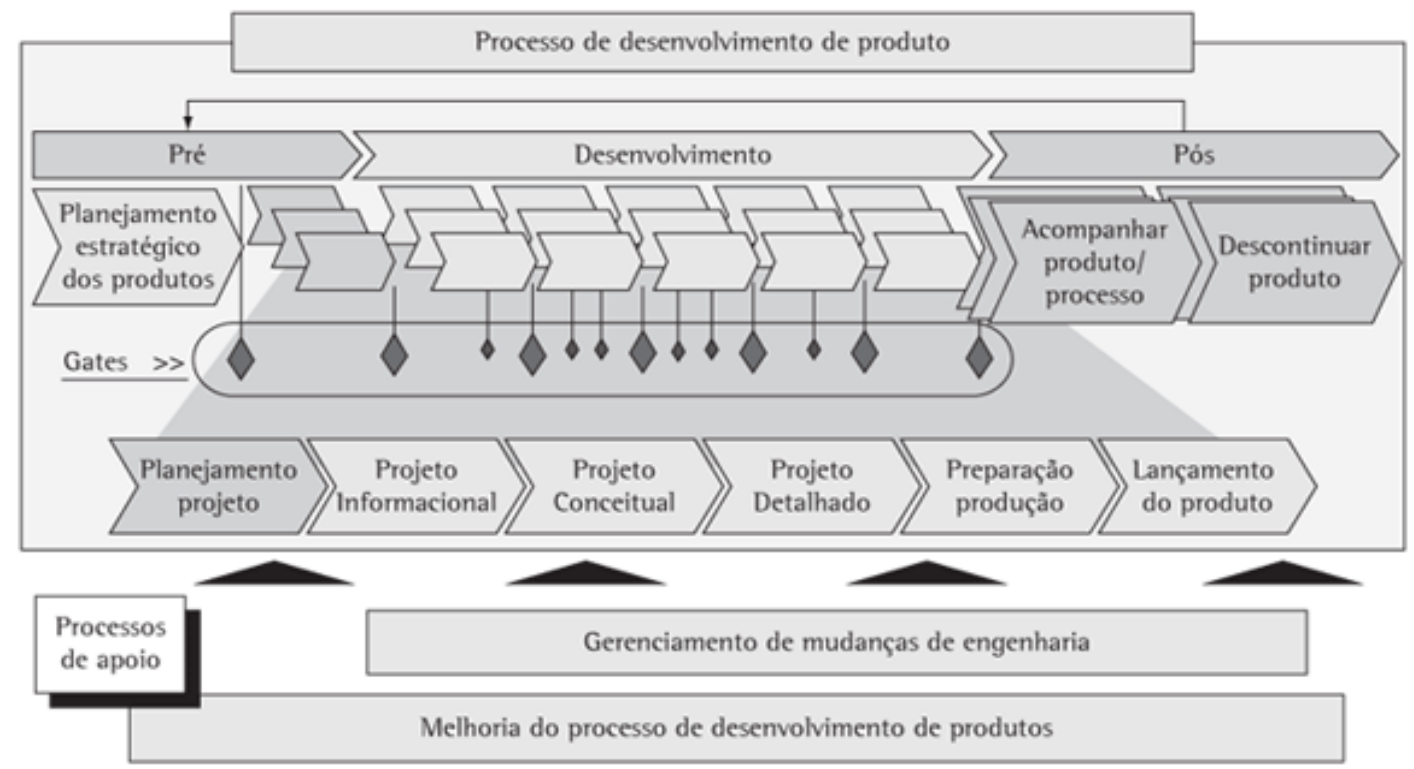

O trabalho em questão está inserido na fase de desenvolvimento do produto. Por meio das entrevistas realizadas com pessoas que apresentam paralisia em punho e mão, identificaramse as principais especificações que o produto final deverá possuir. Dessa maneira, o conceito do produto a ser desenvolvido foi definido passando para a fase seguinte de projeto detalhado, a qual engloba a modelagem e a impressão 3D do produto de TA. As fases seguintes são oportunidades de pesquisa futuras.

\section{PRODUTOS DE TECNOLOGIA ASSISTIVA}

Tecnologia assistiva (TA) é um termo utilizado de forma genérica para designar um conjunto de tecnologias, produtos, serviços e sistemas utilizados por idosos, ou portadores de deficiências diversas para promover a independência e participação na sociedade e/ou habilitar a realização de atividades que, de outra forma, poderiam ser difíceis, oferecer perigo ou ser impossíveis de executar (AUDIT COMMISSION, 2004; EUROPEAN COMMISSION, 2003; ISO, 2011).

Radabaugh (1993) define que para as pessoas sem deficiência, a tecnologia torna as coisas mais fáceis. Para as pessoas com deficiência, no entanto, a tecnologia torna as coisas possíveis. De acordo com Normann (2006), a maioria das tarefas da vida diária é rotineira e exige pouco pensamento ou planejamento, já as atividades não cotidianas exigem 
considerável planejamento, estratégias de tentativas e erro deliberadas. Entretanto, para as pessoas com deficiência as atividades rotineiras, na vida pessoal, social e escolar, muitas vezes apresentam-se como tarefas que exigem um grau de desempenho maior do que lhes é possível realizar. Neste contexto, a tecnologia assistiva representa um recurso para a otimização das atividades. Assim, a adaptação de utensílios utilizados por pacientes durante a realização de suas atividades diárias poderá de forma proativa elevar os níveis de autonomia, independência e consequente inclusão.

\subsection{Tecnologia Assistiva e Produtos para Limitações das Mãos}

Em relação aos produtos de tecnologia assistiva que apoiam a realização de atividades com as mãos, Rodrigues Junior, Muniz e Xavier (2015) apresentam uma adaptação funcional para melhorar o desempenho de hansenianos com mão em garra durante a alimentação. O enfoque está em demonstrar a autopercepção dos pacientes acerca da melhora do seu desempenho no ato de se alimentar. O resultado deste estudo demonstrou o aumento do grau de independência durante a realização das atividades de vida diária, mostrando significância estatística ao comparar os resultados obtidos antes e depois do uso das adaptações. No entanto, este estudo não apresenta um processo de desenvolvimento de produto, assim como não faz uso da tecnologia de impressão 3D.

Outro dispositivo, criado por Finch, Finch e Cook (2016), aborda a questão da manutenção de peso e da força de aperto das mãos. Este dispositivo foi desenvolvido, na área médica, para ajudar os indivíduos que sofrem com a fraqueza de aderência da mão por uma doença, como a artrite. Desta forma, o dispositivo é usado na mão para facilitar a retenção de qualquer peso e para reduzir a necessidade de força de agarramento, podendo ser usado para suportar bebidas ou qualquer outro recipiente.

O estudo de Kjeven (2013) explora estratégias de autogestão para indivíduos com ósteoartrite nas mãos. Para tanto realiza entrevistas com estes pacientes e conclui que há uma grande variedade de estratégias de autogestão para suportar o desempenho das atividades diárias, nas quais o uso de dispositivos assistenciais e a adaptação das atividades são as estratégias mais frequentemente relatadas.

As dificuldades enfrentadas por pacientes que possuem limitações ao realizar atividades com as mãos, identificadas nestes estudos, evidenciam que o desenvolvimento de um produto para apoiar a realização das atividades diárias é eficaz no objetivo de proporcionar a melhoria da qualidade de vida dos pacientes.

\section{MANUFATURA ADITIVA}

A Manufatura Aditiva (MA) ou impressão 3D vem sendo utilizada desde os anos 90 com o intuito de produzir protótipos relativamente rápidos para a inspeção visual, avaliação ergonômica e análise da forma, e assim auxiliar no processo do desenvolvimento de um produto (CHOI; CHAN, 2005). O termo manufatura aditiva é uma designação genérica de uma variedade de tecnologias que podem ser usadas para a fabricação de modelos físicos diretamente a partir de um modelo CAD 3D, usando processos aditivos (PIPES, 2010).

Com esse processo, torna-se possível transformar modelos digitais com geometrias complexas em artefatos físicos, sendo este considerado "aditivo", por combinar camadas de material polimérico para se criar um objeto sólido (GORNI, 2001).

De acordo com Gorni (2001), todos os processos de manufatura aditiva atualmente existentes são constituídos por cinco etapas básicas: 
a) criação de um modelo CAD da peça projetada;

b) conversão do arquivo CAD em formato STL;

c) fatiamento do arquivo STL em finas camadas transversais;

d) construção física do modelo, empilhando-se uma camada sobre a outra;

e) limpeza e acabamento do protótipo.

Dentre as mais variadas vantagens da impressão 3D estão: construção de protótipos precisos em tempos bem inferiores aos obtidos com métodos tradicionais; redução de custos e riscos de desenvolver produtos e ferramentas antes de receber a aprovação de seus clientes; validação visual para desenvolvimento de ferramentas; impressão das mais complexas geometrias e pequenos detalhes; facilidade de duplicação dos modelos a custo reduzido; cópias podem ser feitas em várias escalas; e a minimização de problemas de engenharia como encaixes e montagens (SANTOS, 2011).

\section{ASPECTOS METODOLÓGICOS}

A metodologia adotada para o desenvolvimento do produto assistivo é similar à metodologia para o desenvolvimento de qualquer outro produto, com a particularidade de enfocar nas necessidades de um usuário específico. Desta maneira, foi utilizado como base para esta pesquisa o Processo de Desenvolvimento de Produto proposto por Rozenfeld et al. (2006). A particularidade deste projeto é atendar as necessidades específicas de indivíduos portadores de paralisia muscular na mão e punho, os quais possuem dificuldades de realizar algumas funções básicas.

Inicialmente realizou-se um levantamento na literatura acerca da temática envolvida por esta pesquisa. Assim, foi realizada uma avaliação das tecnologias existentes no Catálogo Nacional de Produtos de Tecnologia Assistiva (MCTI, 2015) e percebeu-se a ausência de produtos versáteis que auxiliem na solução de mais de um problema. Ou seja, um único produto de tecnologia assistiva que possa ser utilizado pelo paciente para a realização de diversas atividades.

A partir de informações obtidas por meio do levantamento na literatura, de entrevistas realizadas com pessoas que apresentam as limitações supracitadas e de um brainstorming foi então definido um produto que atende as necessidades do usuário.

Durante a etapa de definição do produto, concluiu-se que o projeto deveria ser de material simples, de baixo custo, de fácil utilização, ajustável para diversos tamanhos de mãos e que apresentasse segurança e firmeza ao usuário na utilização para atividades do dia a dia, com enfoque no ato de se alimentar e se higienizar. Com isso, identificou-se que o produto deveria ser elaborado a partir de um material polimérico, com um furo imantado na extremidade para permitir que objetos distintos (garfo, faca, pente, entre outros), compostos por aço inoxidável, se acoplem com firmeza ao produto. Além disso, priorizou-se a praticidade na limpeza. Por fim, definiu-se que o produto deveria apresentar alças para possibilitar o uso de velcro, o qual permitirá o ajustamento do dispositivo de acordo com o tamanho e a geometria da mão do usuário.

Com base nessas informações, foi realizada a digitalização da mão e punho de um indivíduo por meio do programa 123D Catch (Autodesk Inc.). A partir da digitalização, o produto foi modelado no programa Solidworks com a finalidade de realizar uma visualização tridimensional, realizar ajustes, revisar as medidas e elaborar um layout que corroborasse com as características almejadas no brainstorming. Outros pontos a serem considerados é que o modelo CAD obtido permite que hajam manipulações das formas e volumes relacionados à 
ergonomia e as proporções do corpo humano, permitindo assim, uma maior observação aos detalhes, e estudo de dobras, linhas, estruturas e volumes.

Por fim, foi criado um mockup do produto por intermédio da impressão 3D. Esta etapa foi desenvolvida em uma impressora 3D Cloner DH+, a qual utiliza como material o PLA (Ácido Poliláctico). Este material é um filamento plástico constituído de material biodegradável, gerado a partir de fontes naturais como milho. Apresenta um ótimo resultado no processo de impressão. A espessura utilizada na impressora 3DCloner foi de $0,25 \mathrm{~mm}$.

\section{RESULTADOS}

\subsection{Particularidades do Processo de Desenvolvimento de Produtos Assistivos}

Avaliando o Processo de Desenvolvimento de Produto do trabalho em questão, é possível realizar algumas comparações com o PDP dos demais produtos. Primeiramente, pode-se afirmar que os produtos assistivos são criados para atender somente um ou poucos usuários específicos os quais possuem limitações que devem ser profundamente levadas em consideração em todas as etapas de desenvolvimento do produto. Além disso, uma vez que o produto deverá ser utilizado para auxiliar o indivíduo na realização de atividades básicas, podendo inclusive substituir a utilização de um membro do corpo, deve-se levar em consideração os fatores ergonômicos de cada pessoa para que não haja nenhum incômodo ao usuário e posterior abandono do produto.

Percebe-se também que para o desenvolvimento de um produto assistivo há um maior tempo investido nas etapas de projeto informacional, conceitual e detalhado do que no desenvolvimento de produtos tradicionais. Tal fato se deve à importância que deve ser dada para se compreender com bastantes detalhes as deficiências do usuário, suas características físicas e o perfil de uso do produto a ser desenvolvido. Outro ponto que chama a atenção é a maior participação do usuário em todas as etapas do PDP.

\subsection{Proposta de produto}

O produto assistivo proposto é composto por um dispositivo de apoio e utensílios complementares para que usuários com paralisia na mão e punho possam ter autonomia na realização de atividades diárias, como o ato de se alimentar e se higienizar. Após a digitalização, o dispositivo foi modelado com base no formato ergonômico da mão e dos encaixes dos dedos, local onde deverá ser encaixado. Desta maneira, a pega do dispositivo foi suavizada para que se acople ao formato dos dedos. Nas laterais foram criadas duas alças que permitem o envolvimento de tiras de velcro ao dispositivo para assim possibilitar o ajuste à mão do usuário, bem como conferir a firmeza necessária para evitar a soltura.

Em uma das extremidades do dispositivo, foi criada uma cavidade octogonal, a qual deverá possuir um imã em seu interior para viabilizar o perfeito acoplamento de utensílios complementares Estes utensílios, todos com encaixe octogonal na extremidade, deverão ser elaborados com material ferromagnético e serão partes integrantes do produto assistivo. A princípio foram desenvolvidos utensílios que auxiliam no ato de alimentar, como talheres e no ato de pentear os cabelos. No entanto, podem ser desenvolvidos outros utensílios para as atividades do dia a dia (e.g. objetos que facilitem a limpeza, higienização, objetos de estudo e trabalho, entre outros). Dessa maneira, o usuário não necessita remover o dispositivo de apoio de sua mão para execução de atividades diferentes, bastando apenas trocar o utensílio complementar acoplado à cavidade.

Primeiramente, o dispositivo proposto e seus utensílios complementares foram modelados para estudo da forma e dimensionamento no programa Solidworks. Por fim, o produto 
assistivo é apresentado na forma de um mock-up produzido em PLA, elaborado a partir do modelo construído no CAD, por meio da impressão 3D para que sejam posteriormente realizadas as etapas de avaliação e produção final do produto. Para a fase de estudo do mockup, ressalta-se que foi impresso em 3D apenas o dispositivo de apoio por questões de custo. As figuras 2 e 3 apresentam o produto assistivo proposto, o dispositivo de apoio e seus utensílios complementares respectivamente. Na figura 4 é possível identificar o mock-up final da proposta do produto.

Figura 2: Proposta de produto assistivo para paralisia em punho e mão - dispositivo de apoio. Fonte: Os autores.

Figura 3: Utensílios complementares do dispositivo de apoio. Fonte: Os autores.

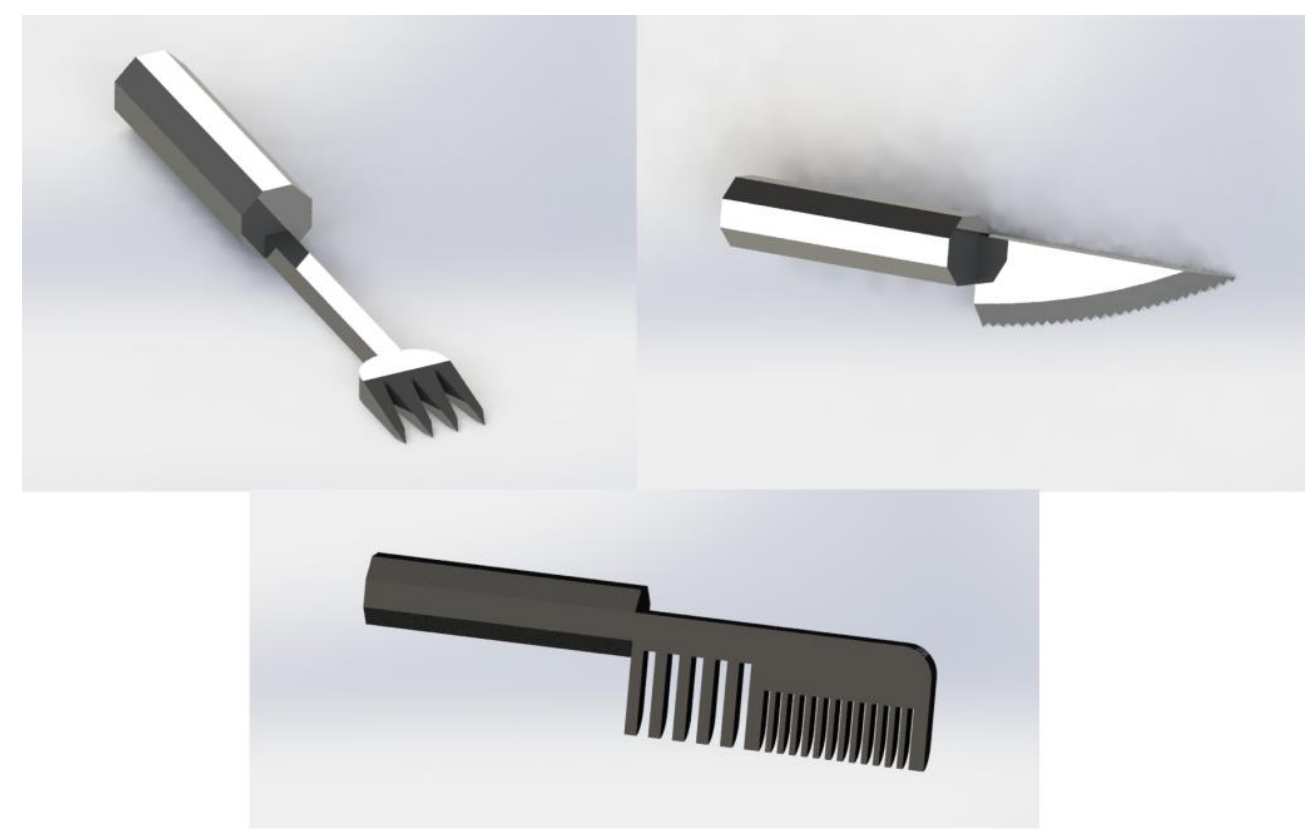




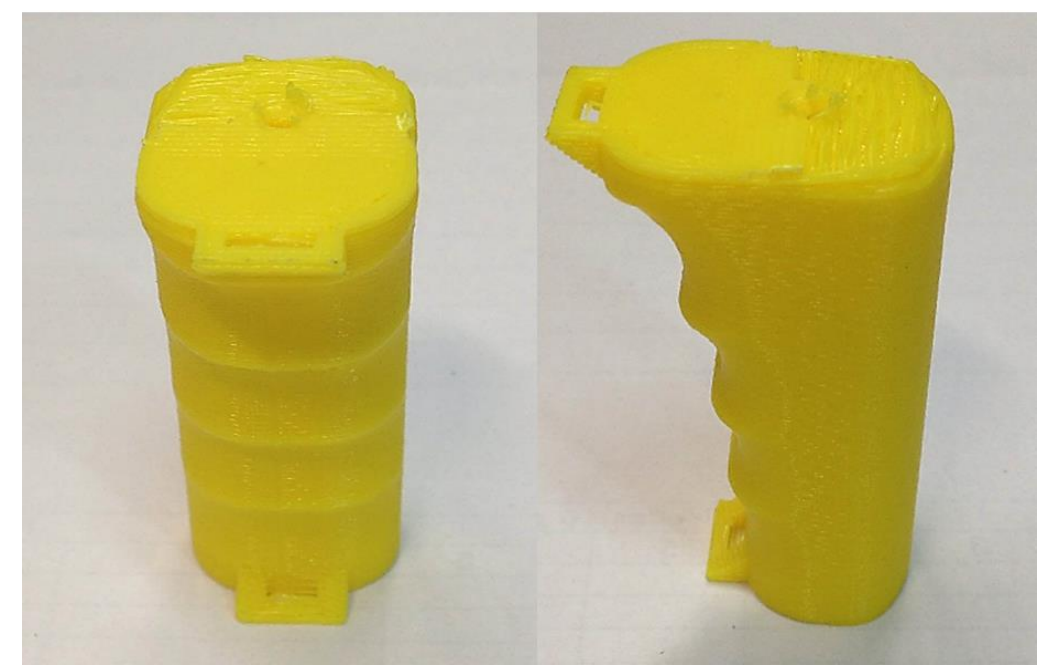

\section{CONSIDERAÇÕES FINAIS}

Por meio da adoção do Processo de Desenvolvimento de Produto tradicional, com a particularidade de enfoque nas necessidades específicas de indivíduos com paralisia no punho e na mão, foi possível alcançar o objetivo desta pesquisa. O produto proposto tem como intuito auxiliar pacientes que se deparam com limitações diárias na realização de diversas atividades funcionais. Este produto é composto de um dispositivo de apoio e utensílios complementares.

A proposta de produto apresentada possui como foco, características de:
a) versatilidade;
b) uso de material simples;
c) baixo custo;
d) facilidade de utilização;
e) segurança e firmeza.

Ressalta-se ainda que o processo de impressão 3D viabilizou a criação de um protótipo em tempo reduzido e com custos inferiores quando comparado aqueles obtidos por métodos tradicionais. Desta maneira, conclui-se que este processo pode proporcionar uma redução nos custos de projeto final, uma vez que possibilita quaisquer alterações no projeto do produto ainda em suas etapas iniciais.

Esta pesquisa pretende proporcionar ao usuário a autonomia necessária para o alcance da melhoria de qualidade de vida, a qual é reflexo do aumento da autoconfiança, da melhoria de inserção social e até mesmo da melhoria dos resultados de tratamentos coadjuvantes. Além disso, este estudo permite o acesso à informação sobre a tecnologia assistiva, bem como apresenta uma comparação entre o processo de desenvolvimento de produto tradicional e de produto assistivo. Menciona-se ainda como contribuição deste estudo o favorecimento à reflexão sobre o tema de inclusão dos pacientes com paralisia do punho e da mão.

Como etapas futuras desta pesquisa, determina-se que após a análise preliminar do mock-up do produto desenvolvido, este será validado junto aos usuários para que então possa ser produzido em sua versão final. 


\section{REFERÊNCIAS}

ASIMOV, M. Introduction to design: fundamentals of engineering design. [S.1.]: Prentice Hall, 1962.

AUDIT COMMISSION. Older people and well-being: the challenge for public services. $2004 . \quad$ Disponível em: $<$ http://archive.auditcommission.gov.uk/auditcommission/sitecollectiondocuments/AuditCom misionReports/NationalStudies/OlderPeople_overarch.pdf>. Acesso em: Maio, 2017.

BACK, N. Metodologia de projeto de produtos industriais. Rio de Janeiro: Guanabara Dois, 1983.

BORG, J.; LARSSON; S.; ÖSTERGREN, P-O. The right to assistive technology: for whom, for what, and by whom? Disability \& Society, v. 26, n. 2, p. 151-167, 2011.

BUSS, C. de O.; CUNHA, G. D. da. Modelo referencial para o processo de desenvolvimento de novos produtos. Anais. XXII Simpósio de Gestão da Inovação Tecnológica. Salvador, Bahia, 2002.

CHOI, S.H.; CHAN, A.M.M. A virtual prototyping system for rapid product development. Computer-Aided Design, v. 36, p. 401-412, 2004.

EUROPEAN COMMISSION. Access to assistive technology in the European Union. 2003. Disponível em: <http://www.acessibilidade.net/at/access_AT_EU.pdf>. Acesso em: Maio, 2017.

FINCH, T. R.; FINCH, P. D.; COOK, D. R. Assistive apparatus for hand held vessels or items. U.S. Patent n. 9,237,796, 19 jan. 2016.

GORNI, A. A. Introdução à prototipagem rápida e seus processos. Revista Plástico Industrial, p. 230-239, mar. 2001.

INTERNATIONAL ORGANIZATION FOR STANDARDIZATION - ISO. ISO 9999. Assistive products for persons with disability. Classification and terminology. 2011. Disponível em: <http://www.iso.org/iso/home/store/catalogue_ics/catalogue_detail_ics.htm?csnumber=50982 >. Acesso em: Maio, 2017.

KJEKEN, I. et al. Self-management strategies to support performance of daily activities in hand osteoarthritis. Scandinavian journal of occupational therapy, v. 20, n. 1, p. 29-36, 2013.

KRISHNAN, V.; ULRICH, K. T. Product Development Decisions: a Review of the Literature. Management Science, v. 47, n. 1, p. 1-21, 2001.

MINISTÉRIO DA CIÊNCIA, TECNOLOGIA E INOVAÇÃO. Catálogo Nacional de Produtos de Tecnologia Assistiva. Disponível em : < http://assistiva.mct.gov.br/>. Acesso em : 02/2017.

NORMANN, D. A. O design do dia-a-dia. Rio de Janeiro: Rocco, 2006.

ØIEN, I.; FALLANG, B.; ØSTENSJØ, S. Everyday use of assistive technology devices in school settings. Disability Rehabilitation Assistive Technology, Early Online: 1-6, 2015.

PAHL, G. et al. Projeto na Engenharia: Fundamentos do desenvolvimento eficaz de produtos, métodos e aplicações. São Paulo: Edgard Blücher, 2005. 
PAHL, G.; BEITZ, W. Engineering Design: A Systematic Approach. [S.1.]: Editora Springer Verlag, 2003.

PAULA, F.V.R.; FARIA, C.D.C.M.; VIEIRA, D.S.R. Teoria da programação motora: uma perspectiva da sua evolução teórica. Revista Fisioterapia em Movimento, Curitiba, v. 20, n. 2, p. 63-71, 2007.

PENG, D. X.; HEIM, G. R.; MALLICK, D.N. Collaborative Product Development: The Effect of Project Complexity on the Use of Information Technology Tools and New Product Development Practices. Production and Operations Management, v. 23, n. 8, p. 1421-1438, 2014.

PIPES, A. Desenho para Designers. São Paulo: Editora Blucher, 2010.

RADABAUGH, M.P. Selecting and obtaining assistive technology - IBM national support center for persons with disabilities. In: Interagency Project for assistive technology. North Dakota: IPAT, 1993.

RODRIGUES JUNIOR, J. L.; MUNIZ, L. S.; XAVIER, M. B. A utilização da tecnologia assistiva para alimentação na melhora do desempenho ocupacional de hansenianos com mão em garra. Hansen Int, v. 39, n. 1, p. 22-29, 2015.

ROZENFELD, H. et al. Gestão de Desenvolvimento de Produtos: Uma referência para a melhoria do processo. [S.1.]: Editora Saraiva, 2006.

SANTOS, S.N.S. Curso Nacional de Aprendizagem Industrial-SENAI - Impressora 3D. SENAI, 2011. 